\title{
Necroptosis of osteoblasts was induced by breast cancer cells in vitro
}

\author{
Xiang Ji, Ruideng Wang, Hai Tang, Hao Chen, Li Bao, Fei Feng, Pu Jia \\ Department of Orthopedics, Beijing Friendship Hospital, Capital Medical University, Beijing 100050, China \\ Contributions: (I) Conception and design: X Ji, P Jia; (II) Administrative support: H Tang; (III) Provision of study materials or patients: H Chen; (IV) \\ Collection and assembly of data: L Bao; (V) Data analysis and interpretation: F Feng; (VI) Manuscript writing: All authors; (VII) Final approval of \\ manuscript: All authors. \\ Correspondence to: Pu Jia. Department of Orthopaedics, Beijing Friendship Hospital, Capital Medical University, No. 95, Yong'an Road, Xicheng \\ District, Beijing 100050, China. Email: jiapu@ccmu.edu.cn.
}

\begin{abstract}
Background: Bone metastasis of breast cancer could lead to serious osteolysis and severe pain. This study is aimed to investigate the existence of necroptosis, a new type of programmed cell necrosis pathway, in breast cancer-induced osteoblast cell death.

Methods: In this study, conditioned medium (CM) of breast cancer cells was prepared to simulate the micro-environment of bone metastasis in breast cancer in vitro and co-cultured with osteoblast. Then the percentage of cell survival and death was detected via cell viability and flow cytometry. Western blot and PCR were taken to measure protein and mRNA expression level of RIPK 3, MLKL and caspase 3 respectively.

Results: CM could induce osteoblasts death, including apoptosis and necroptosis and necrostatin-1 plus Z-IETD-FMK could decrease the percentage of death cells significantly in the flow cytometry detection. Moreover, CM could increase cleaved caspase 3, RIPK 3 and p-MLKL significantly, while RIPK 3 and p-MLKL was reduced statistically when osteoblasts were treated with Necrostatin-1 (Nec-1). In addition, the mRNA level of three proteins was not consistent with the change of their corresponding protein level.

Conclusions: In conclusion, the necroptosis pathway exists in osteoblast cell death pathway induced by breast cancer cells and could be inhibited by Necrostatin-1 (Nec-1).
\end{abstract}

Keywords: Breast cancer bone metastasis; osteoblast; necroptosis; necrostatin-1; co-culture system; RIPK 3; MLKL; Caspase 3

Submitted Aug 08, 2019. Accepted for publication Nov 11, 2019.

doi: $10.21037 /$ tcr.2019.11.32

View this article at: http://dx.doi.org/10.21037/tcr.2019.11.32

\section{Introduction}

Bone tissue is one of the most common metastasis sites of malignant tumors, and about $70-80 \%$ of patients with malignant tumors eventually develop bone metastasis $(1,2)$. Among these malignant tumors, breast cancer is one of the highest morbidity and mortality around the world and its most common distal metastasis site is bone tissue, of which about $70 \%$ patients had a confirmed bone metastasis in autopsy (3). In clinical observation, bone metastasis of breast cancer is usually osteolytic, and often leads to destruction and dissolution of bone, as well as cause severe pain to patients' physiology and psychology, which seriously reduces the patients' quality of life. Therefore, the research of bone metastasis mechanism breast cancer has always been a hot topic.

In 1989, Stephen Paget (4) proposed the hypothesis, called "seed and soil", that the interaction between tumor cell and particular organ micro-environment determined the preferential selection of cell growth. Moreover, Mundy (5) suggested that the pathological changes of bone metastasis in breast cancer were mainly osteolysis and destruction of bone structure mediated by osteoclast in his study. It 
resulted from that the bone metastasis of breast cancer often disturbs the balance of osteoblasts and osteoclasts, which increased osteoclasts activity and accelerate bone resorption. Currently, most studies about the mechanism of bone metastasis of breast cancer focus on the interaction between osteoclast and breast cancer cells, while there are few studies on the interaction between breast cancer cells and osteoblast. Mercer (6) created the conditioned medium (CM) of MDA-MB-231 as simulated micro-environment of bone metastasis in breast cancer and used it to culture with MC3T3-El cells, which downregulated some important proteins related to osteoblast differentiation. In addition, Mastro (7) found that breast cancer cells could increase osteoblast apoptosis through co-culture. The pathway of osteoblast apoptosis induced by breast cancer cells and the interaction between osteoblasts and breast cancer cells become more popular recently.

Necroptosis is a new type of cell death pattern with necrosis morphological characteristics, which is regulated and non-caspase-dependent programmed cell necrosis, as well often accompanied by cell lysis and inflammation (8). It begins with the binding of death inducible factor-related ligands with their corresponding receptors to form complex I, then the deubiquitized RIP1 (receptor interaction protein kinase 1) separates from complex I and recruits complex II containing RIP3 (receptor interaction protein kinase 3) and caspase-8 (9). When caspase 8 is inhibited, phosphorylation of RIPK3 is initiated, which starts the necroptosis process and results in morphological changes of cell necrosis (10). As a specific inhibitor of necroptosis pattern, Necrostatin-1 (Nec-1) can inhibit the activation of RIP 1 to affect expression of its downstream molecule RIP 3 (11). On the contrary, if a cell necrosis process can be blocked by Nec-1, it can be confirmed that the mode of cell death is necroptosis. Recently, numerous studies have confirmed the existence of necroptosis in a series of diseases by means of Nec-1 blockade and RIP3 gene knockout, such as neurodegenerative diseases (12), ischemic diseases (13), inflammation and infection $(14,15)$. Moreover, some studies have confirmed that breast cancer cells can lead to osteoblast apoptosis, but whether breast cancer cells can lead to necroptosis of osteoblast has not been reported.

In this study, the co-culture system of breast cancer cells and osteoblasts in vitro is constructed to simulate the osteolytic micro-environment, and some tests are taken to observe whether breast cancer cells could induce necroptosis of osteoblasts.

\section{Methods}

\section{Cell culture and chemicals}

The MC3T3-E1 osteoblasts cell line and the 4T1 mouse breast cancer cell line were provided by the National Infrastructure of Cell Line Resource (China). Necrostatin-1 (Nec-1, 4311880, USA), the specific inhibitor of RIPK 1, was provided by MedChemExpress (USA). The specific inhibitor of caspase 8, named Z-IETD-FMK (S7314; USA), was purchased from Selleck (USA). These two reagents are soluble in DMSO (Methyl sulfoxide, 67-68-5, Sigma, USA), then were added medium to adjust concentration to 50 and $40 \mu \mathrm{M}$ for subsequent experiments respectively. The primary antibodies of RIPK3 (ab56164) and p-MLKL (ab196436) were purchased from Abcam (UK), and the primary antibodies of cleaved caspase 3 (9661s), caspase 3 (9662s) and MLKL (37705S) were got from Cell Signaling Technology (USA). $\beta$-actin (TA-09) and goat anti-mouse/ rabbit IgG secondary antibodies were obtained from ZSGBBIO (China).

The MC3T3-E1 cells was incubated in $\alpha-M E M$ medium (32571036; Gibco, USA), which contained 1\% penicillin/streptomycin (15140-122; Gibco, USA), 10\% fetal bovine serum (10099-141; Gibco, Australia) and glutamine, in a constant temperature incubator with $5 \%$ $\mathrm{CO}_{2}$ and $37^{\circ} \mathrm{C}$.

\section{CM}

4T1 cells, a mouse breast cancer cell line, were grown in 1640 medium (RNBH6756, Sigma, UK) in a constant temperature incubator with $5 \% \mathrm{CO}_{2}$ and $37{ }^{\circ} \mathrm{C}$, and then the cells were inoculated into $100 \mathrm{~mm}$ dishes. When reaching $80 \%$ confluence, the medium was removed and cells were rinsed with phosphate-buffered saline (PBS, P1020-500, Solarbio, USA). After that, $5 \mathrm{~mL}$ serum-free 1640 medium was added to culture with the cells for $24 \mathrm{~h}$, and then centrifuged for 5 minutes at $1,000 \mathrm{r} / \mathrm{min}$. The supernatant was collected, aliquoted and stored at $-20^{\circ} \mathrm{C}$ for future use.

This experiment was divided into five groups: blank control group; CM group; CM plus Z-IETD-FMK $(40 \mu M)$; CM plus Nec-1 $(50 \mu M)$; CM plus Z-IETD-FMK $(40 \mu \mathrm{M})$ and Nec-1 $(50 \mu \mathrm{M})$. This study mainly involves cell experiments, in which all of the cells used are purchased from the National Infrastructure of Cell Line Resource (China), and none of animals or human is involved in this study, so the Ethics Approval is not required. 


\section{Cell viability}

In order to the CCK-8 assay direction, MC3T3-E1 cells were planted in 96-well culture dishes in a constant temperature incubator with $5 \% \mathrm{CO}_{2}$ and $37{ }^{\circ} \mathrm{C}$. When cells reached approximately $80 \%$ confluence, the medium was removed and replaced with $\mathrm{CM}$, which was mixed with serum- $\alpha$-MEM medium according to concentration gradient $(0 \%, 20 \%, 40 \%, 60 \%, 80 \%, 100 \%)$. After cultured for 24, 48 and $72 \mathrm{~h}, \mathrm{CM}$ was removed and replaced by $100 \mu \mathrm{L}$ medium containing $10 \%$ CCK-8 (Dojindo, Japan). It took $3 \mathrm{~h}$ to incubate $\mathrm{MC} 3 \mathrm{~T} 3-\mathrm{E} 1$ cells at $37^{\circ} \mathrm{C}$, and then a microplate reader (Model 680; Bio-Rad Laboratories, USA) was used to measure the absorbance at a wavelength of $490 \mathrm{~nm}$. Cell viability was obtained by the formula of absorbance provided by CCK8 specification.

\section{Cell death assay}

MC3T3-E1 cells were planted in 24-well plates at a density of $2 \times 10^{5} / \mathrm{mL}$. After the cells grew to approximately $80 \%$ confluence, the medium was replaced with fresh medium, and then these cells were dealt with Z-IETD-FMK $(40 \mu M)$, Nec-1 $(50 \mu M)$, Z-IETD-FMK (40 $\mu M)$ plus Nec-1 $(50 \mu \mathrm{M})$ for $6 \mathrm{~h}$, respectively. Next, $\mathrm{CM}$ was added to the plates, in which the concentration of CM reached to $40 \%$, to culture with the cells for $24 \mathrm{~h}$. Subsequently, five groups cells were rinsed with PBS and harvested by trypsinization, and then centrifuged at 3,000 rpm for 5 minutes. After that cells were suspended and stained with Annexin V and PI via FITC Annexin V Apoptosis Kit (MultiSciences Biotech Co., Ltd., China) according to instructions. The proportion of cell survival, apoptosis and necrosis was analyzed via flow cytometry (FACS, BD Biosciences). Moreover, data were the average result of three independent experiments and analysed by FlowJo VX software.

\section{Western blot analysis}

MC3T3-E1 cells were obtained through the same process as described in cell death assay part. Instead of harvesting by trypsinization, the cells were lysed in Radioimmunoprecipitation assay (RIPA) lysis buffer containing $4 \%$ phosphorylase inhibitors, $4 \%$ protease inhibitors, $4 \% \mathrm{PMSF}$ for $30 \mathrm{~min}$ at $4{ }^{\circ} \mathrm{C}$. After centrifugation at 3,000 rpm for $10 \mathrm{~min}$, the supernatants were aspirated and their protein concentrations were determined by BCA assay. The equivalent amount of protein lysates was separated via sodium dodecyl sulfatepolyacrylamide gel electrophoresis (SDS-PAGE) and then transferred onto a polyvinylidene fluoride membrane (Bio-Rad Laboratories) which was incubated in blocking buffer ( $5 \%$ non-fat milk powder in TBST) for $2 \mathrm{~h}$. Next the membranes were incubated overnight at $4{ }^{\circ} \mathrm{C}$ with primary antibody (diluted 1:500) and then incubated with a secondary antibody at room temperature for $2 \mathrm{~h}$. The excess secondary antibody was washed away by using TBST. The membranes were visualized with enhanced chemiluminescence (GE Healthcare, USA). The results were presented in the ratio of expression relative to $\beta$-actin.

\section{Quantitative real-time polymerase chain reaction $(q R T$ - PCR) analysis}

MC3T3-E1 cells were treated as the same process as described above, and Trizol reagent (Sigma, USA) was used to extract the total RNA. Roche reagent (Transcriptor cDNA Synthesis Kit 1, USA) was used to synthesize the cDNA. The qRT-PCR detection was taken via a C1000 thermocycler and an ABI7500 real-time PCR system (Applied Biosystems, USA) with a Roche Fluorometric Quantitation Kit [FastStart Universal SYBR Green Master (ROX)]. The amplification products were determined through amplification curve analysis. The formula method $\left(2^{-\Delta \Delta C T}\right)$ was used to analyze all data, and all these data were standardized to the house-keeping gene $\beta$-actin. Table 1 shows Sequence of primer pairs

\section{Statistical analysis}

The measurement data is expressed as means \pm standard deviations (SD, $\bar{x} \pm s)$ and analyzed statistically via SPSS 22.0 software (SPSS, Chicago, IL, USA) and GraphPad Prism6 software. The unpaired Student's $t$-test was performed to determine the differences among groups. Statistical significance was determined at $\mathrm{P}$ value $<0.05$.

\section{Results}

\section{Effects of 4T1 CM on osteoblasts growth}

After MC3T3-E1 cells cultured with CM in a concentration gradient $(0 \%, 20 \%, 40 \%, 60 \%, 80 \%$, and $100 \%)$ for 24 h, 48 h, 72 h (as shown in Figure 1), the cell viability significantly decreased as the concentration increased at the three time points respectively. In the other hands, when 
Table 1 Sequence of primer pairs

\begin{tabular}{ll}
\hline Gene & Sequence of primer pairs \\
\hline$\beta$-actin & 5'-GGCTGTATTCCCCTCCATCG-3' \\
& 5'-CCAGTTGGTAACAATGCCATGT-3' \\
RIPK3 & 5'-GAACTGAAGAAGCTGGAGTTTGTG-3' \\
& 5'-ATCTTGACTGCTACATCATGGTTCC-3' \\
MLKL & 5'-AATTGTACTCTGGGAATTGCCA-3' \\
& 5'-CTCCAAGATTCCGTCCACAG-3' \\
Caspase 3 & 5'-TGGTGATGAAGGGGTCATTTATG-3' \\
& 5'-TCGGCTTTCCAGTCA-GACTC-3' \\
\hline
\end{tabular}

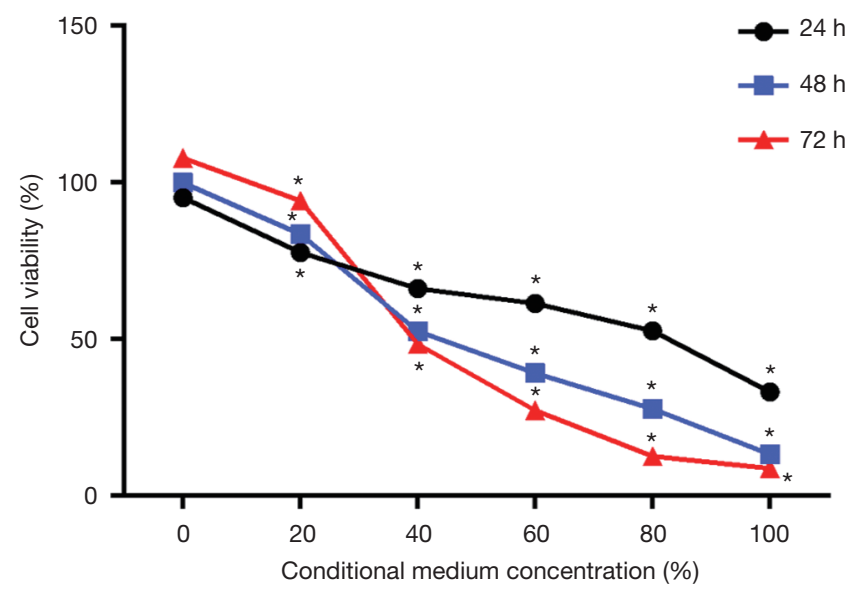

Figure 1 Cell viability of MC3T3-E1 cells was measured using the CCK- 8 assay. Data are reported as means \pm SD of five independent experiments. * means the $\mathrm{P}$ value $<0.05$.

cells were treated with $0 \%$ and $20 \%$ CM, the viability increased over time. However, treatment with more than $40 \%$, CM significantly reduces cell viability over time. These results indicated that CM inhibited MC3T3-E1 cells viability in a dose-dependent manner, and when the percentage of $\mathrm{CM}$ was higher than a certain concentration, the inhibition of $\mathrm{CM}$ was also in a time-dependent manner.

\section{Flow cytometric analysis of cell apoptosis and necrosis}

According to the result of CCK-8, $40 \%$ CM was selected to treat MC3T3-E1 cells for $24 \mathrm{~h}$, then the apoptosis and necrosis were detected via flow cytometric analysis, in which $\mathrm{A}-/ \mathrm{P}$ - standed for cell survival, $\mathrm{A}+/ \mathrm{P}-$ represent apoptosis and $\mathrm{A}+/ \mathrm{P}+$ indicated necrosis. As shown in
Figure 2, treatment with CM (graph B) increased the percentage of cell apoptosis and necrosis compared with control group, which was consistent with the results of CCK- 8 and suggested that the $40 \%$ CM inhibited cell proliferation and induced cell apoptosis and necrosis. After treated with Z-IETD (group D), the special inhibitor of caspase 8 in apoptosis pathway, cells apoptosis was decreased dramatically in comparison with CM group, but the percentage of necrosis was increased compared with $\mathrm{CM}$ and control group. In Nec-1 group (graph C), cell survival ratio was less than the other three groups, and when compared with $\mathrm{CM}$ and $\mathrm{CM}+\mathrm{Z}$-IETD groups, the necrosis percentage was reduced but the apoptosis ratio was increased, which suggested that necrosis pathway could be inhibited by Nec-1, but that would effect the apoptosis pathway. When treated with CM, Z-EITD and Nec-1 (graph E), cells survived more than the other four groups but less then control group. Comparing the apoptosis percentage between five groups, it was decreased in $\mathrm{CM}+\mathrm{Z}$-EITD + Nec-1 group than $\mathrm{CM}$ and $\mathrm{CM}+$ Nec-1 group but increased slightly than CM + Z-EITD group. Moreover, the necrosis percentage in this group was declined obviously. The changes of survival, apoptosis and necrosis percentages were shown in graph F, G and H, which is described as above.

\section{Expression of proteins related to necroptosis pathway}

RIPK 3 is a specific protein in the necroptosis pathway, and Nec-1 as a specific inhibitor of RIPK 1 is able to downregulate the expression of its downstream RIPK 3. As shown in Figure 3 (graph A, B and C), the expression of RIPK 3, p-MLKL and cleavage caspase 3 proteins were increased significantly in $\mathrm{CM}$ group $(\mathrm{P}<0.05)$. After treated with Z-IETD, cleavage caspase 3 was decreased obviously while RIPK 3 and $\mathrm{p}-\mathrm{MLKL}$ expressed increasingly $(\mathrm{P}<0.05)$. When treated with Nec-1 alone, the expression of RIPK 3 and $\mathrm{p}$-MLKL were declined but cleavage caspase 3 expression increased significantly $(\mathrm{P}<0.05)$. In addition, In the Z-IETD and Nec-1 group, the results shown a significant decrease in the expression levels of all three proteins compared with $\mathrm{CM}$ group $(\mathrm{P}<0.05)$. The results demonstrated that CM plus Z-IETD-FMK induced necroptosis, while $\mathrm{CM}$ plus Nec-1 induced apoptosis.

\section{Expression of $m R N A s$ with corresponding proteins}

As shown in Figure 4, the mRNA expression of RIPK3 $(\mathrm{P}<0.05)$, caspase $3(\mathrm{P}<0.05)$ and MLKL $(\mathrm{P}<0.05)$ were 
A

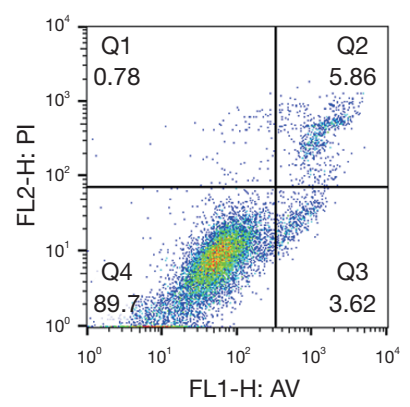

E

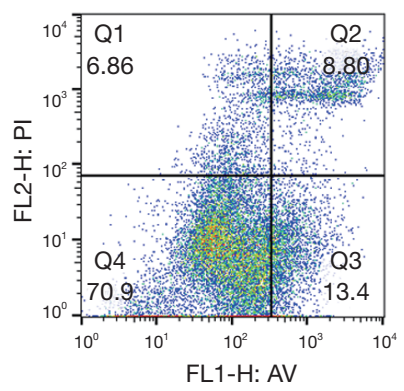

B

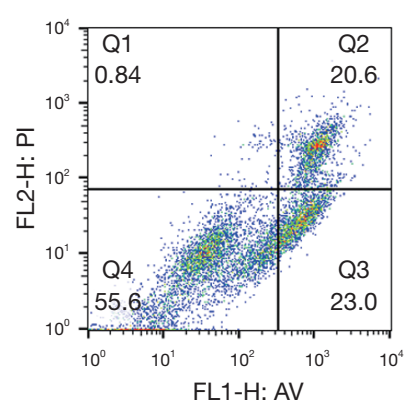

$\mathrm{F}$

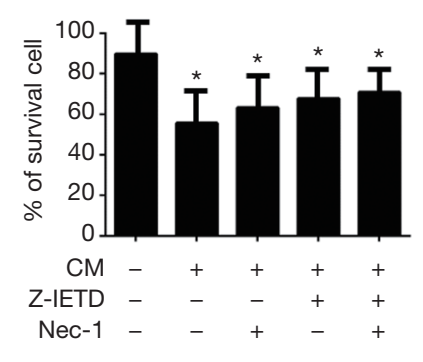

C
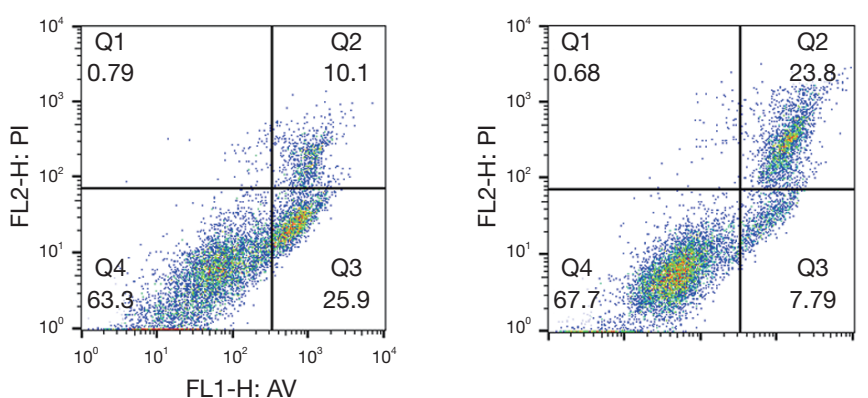

G

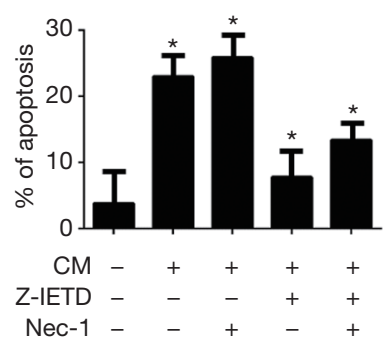

$\mathrm{H}$

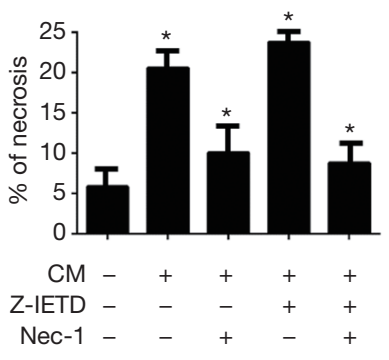

Figure 2 Representative graphs of cell death by flow cytometry analysis after Annexin-V/PI dual staining. Control (A) untreated cells; (B) condition medium (CM) alone; (C) CM + Nec-1; (D) CM + Z-IETD; (E) CM + Z-IETD+Nec-1; (F) cell survival ratio; (G) cell Apoptosis ratio; $(\mathrm{H})$ cell Necrosis ratio. We used the population of A-P- for cell survival, A+P- for apoptosis and A+P+ for necrosis. Data are reported as means $\pm \mathrm{SD}$ of three independent experiments. *, $\mathrm{P}$ value $<0.05$.

significantly increased in CM group. In CM with Nec-1 group, it showed decrease of RIPK3 mRNA expression, but did not show any effect among MLKL and caspase $3 \mathrm{mRNA}$ expression levels. When osteoblast cells were treated with CM and Z-IETD-FMK, RIPK3 mRNA expression was increase but caspase 3 and MLKL mRNA expression were decrease significantly $(\mathrm{P}<0.05)$. In $\mathrm{CM}$ with $\mathrm{Nec}-1$ and Z-IETD-FMK group, it showed significant decrease of RIPK3, MLKL, and caspase 3 mRNA levels $(\mathrm{P}<0.05)$.

\section{Discussion}

As a common type of bone metastatic tumor, bone metastasis of breast cancer could result in osteolytic bone injury and chronic bone pain $(16,17)$. Paget have shown in their studies that the mechanism of osteolysis in breast cancer involves the secretion of cytokines by breast cancer cells that promote the growth and maturation of osteoclasts, as well as cytokines that promote the apoptosis of osteoblasts, thereby disrupting the balance between osteoblasts and osteoclasts, and the accelerated bone resorption leads to osteolysis (4). In addition, Mercer has indicated in their studies that bone metastasis of breast cancer can affect the differentiation and adhesion of osteoblasts, resulting in reducing osteogenesis and accelerating bone loss (6). Moreover, it has been reported by Mastro that co-culture of breast cancer cells with human osteoblast cell line (hFOB1.19) can increase apoptosis of osteoblasts (7).

In this study, mouse breast cancer cell line 4T1 was cultured in vitro to be prepared for CM to co-cultured with mouse osteoblast MC3T3-E1 cell line, which was aimed to simulate the micro-environment of metastatic tumors. According to CCK-8 assay results, it showed that at the same time point, with the increase of the concentration of $\mathrm{CM}$, the cell viability and survival rate was lower, which indicated that the proliferation ability of osteoblasts was concentration-dependent on CM. However, as shown in Figure 1, when the concentration of CM was less than $40 \%$, it had no inhibitory effect on osteoblasts, and the 
A

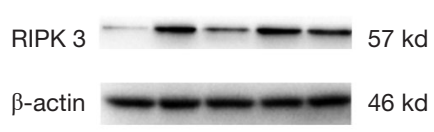

$\begin{array}{rlllll}\text { CM } & - & + & + & + & + \\ \text { Z-IETD } & - & - & - & + & + \\ \text { Nec-1 } & - & - & + & - & +\end{array}$

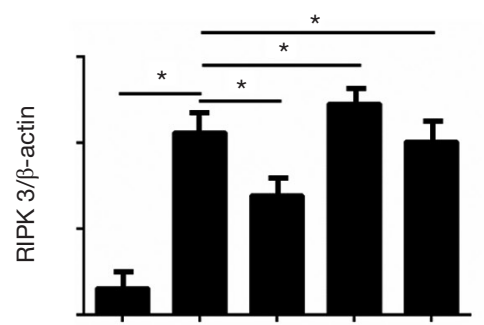

$\begin{array}{rrrrrr}\text { CM } & - & + & + & + & + \\ \text { Z-IETD } & - & - & - & + & + \\ \text { Nec-1 } & - & - & + & - & +\end{array}$

\section{B}
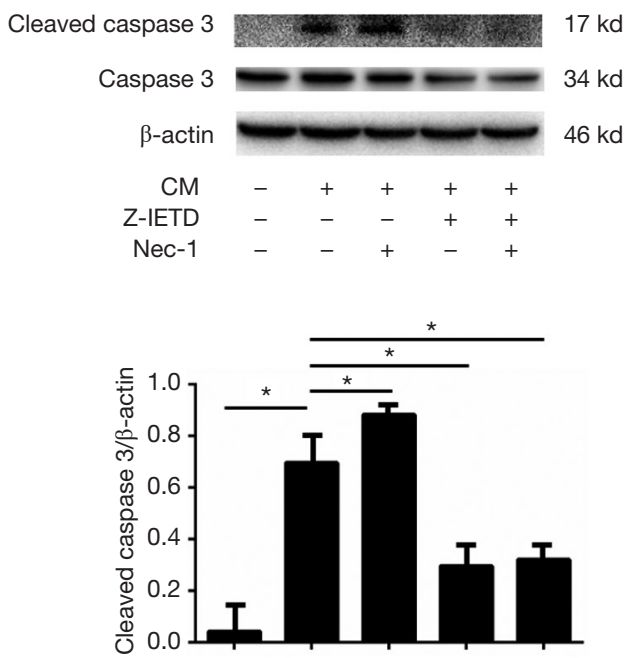

$\begin{array}{rrrrrr}\text { CM } & - & + & + & + & + \\ \text { Z-IETD } & - & - & - & + & + \\ \text { Nec-1 } & - & - & + & - & +\end{array}$
C

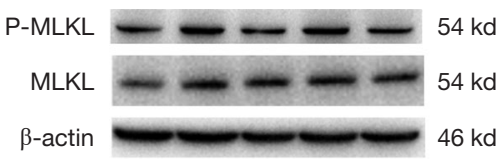

$\begin{array}{rrrrrr}\text { CM } & - & + & + & + & + \\ \text { Z-IETD } & - & - & - & + & + \\ \text { Nec-1 } & - & - & + & - & +\end{array}$

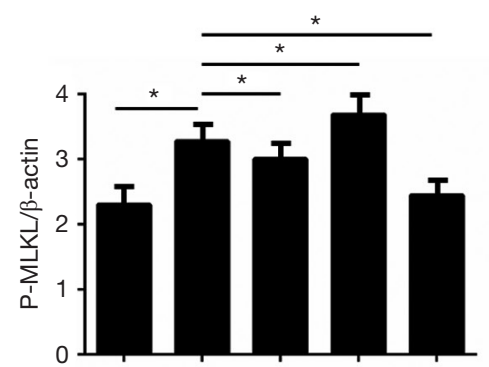

$\begin{array}{rrrrrr}\text { CM } & - & + & + & + & + \\ \text { Z-IETD } & - & - & - & + & + \\ \text { Nec-1 } & - & - & + & - & +\end{array}$

Figure 3 The western blot analysis graphs shows the CM effects on the three proteins expression level in necroptosis and apoptosis pathways. (A) Shows the expression of Specific marker protein RIPK 3; (B) shows the expression of Caspase 3 and cleaved caspase 3; (C) shows the expression of MLKL and p-MLKL. The proportion of target proteins to $\beta$-actin were shown below. Data are reported as means \pm $\mathrm{SD}$ of three independent experiments. *, P value $<0.05$. CM, condition medium.

A

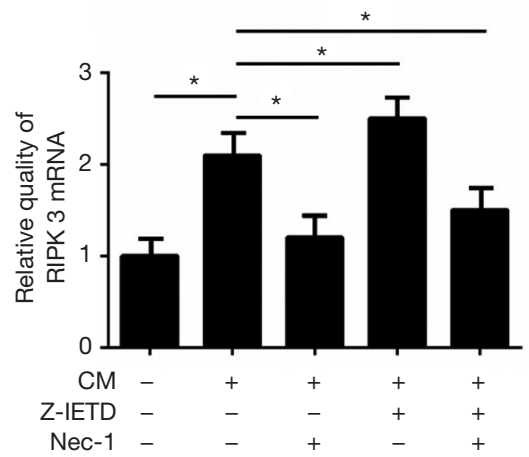

B

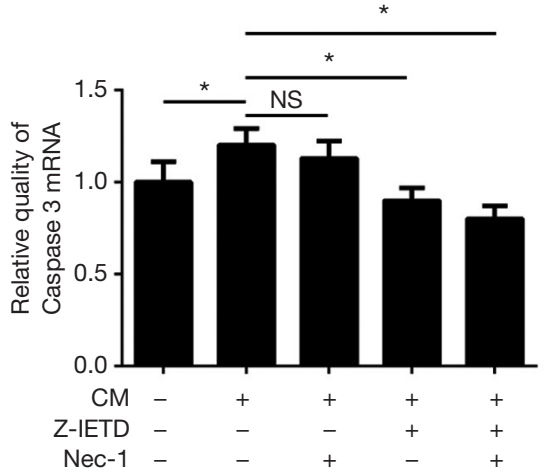

C

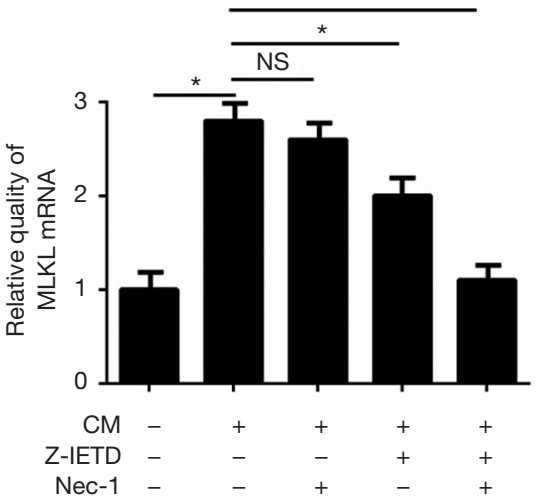

Figure 4 The three proteins corresponding mRNA expression level were detected by qRT-PCR: (A) for RIPK 3; (B) for caspase 3; (C) for MLKL. Data from the treated groups were normalized to $\beta$-actin. Data are reported as means \pm SD of three independent experiments. * $\mathrm{P}$ value $<0.05$. qRT-PCR, quantitative real-time polymerase chain reaction.

survival rate of osteoblasts increased with time. In addition, when the concentration is more than $40 \%$, it inhibited the proliferation of osteoblasts, and the survival rate of osteoblasts was lower with the increases of time. Our study indicated that when the concentration reached a certain level, the breast cancer CM could inhibit osteoblasts over time.

Osteoblasts were co-cultured with $40 \% \mathrm{CM}$ for $24 \mathrm{~h}$ according to the results of CCK-8 experiment, and then detected the apoptosis and necrosis level via flow cytometry 
(Figure 2). It showed the same results with CCK-8 that CM could induce the death of osteoblasts, including apoptosis and necrosis pathways. When we blocked apoptotic pathway with Z-IETD, an apoptotic inhibitors, the proportion of apoptosis was decreased but the necrosis increased. In the other hand, when the necroptosis was blocked by Nec-1, a necroptosis inhibitors, the proportion of necrotic cells decreased significantly compared with CM group, but the proportion of apoptotic cells increased. It indicated that these two pathways may occur simultaneously and have effect on each other under a specific condition, which was also confirmed in Galluzzi et al.'s findings (18).

The RIPK 3 protein, a specific protein in necroptosis pathway, and the Caspase 3 protein in apoptosis pathway were detected by Western blotting test in this study. In the necroptosis forming stage, Nec-1 could inhibit the activity of RIPK 1 to eliminate the formation of compound IIB by RIPK 3 and MLKL protein to inhibit necroptosis pathway $(19,20)$. In addition, Z-IETD could reduce the apoptosis pathway by inhibiting the activation of Caspase 3 into Cleavage Caspase 3 (21). Western blot detection showed that CM could cause the apoptosis and necroptosis after incubation with osteoblasts. Cleavage Caspase 3, a functional protein that induces apoptosis pathway, was effectively inhibited after Z-IETD treatment, but at the same time, RIPK 3 and phosphorylated MLKL protein, the iconic protein of necroptosis pathway, showed significantly increase, which indicated that the necroptotic pathway played a leading role in cell death after the apoptotic pathway was inhibited. On the contrary, when the necroptotic pathway was inhibited by Nec-1 alone, the functional protein Cleavage Caspase 3 in the apoptotic pathway increased significantly, indicating that the apoptotic pathway could be enhanced when the necroptosis pathway was inhibited. When two inhibitors, Nec-1 and Z-IETD, were used together, the effect of inhibiting both pathways can be achieved. Combined with flow cytometry results, we found that the necroptosis pathway, a special pathway of cell necrosis, existed in the co-culture system of breast cancer cells and osteoblasts. Moreover, it showed that apoptosis pathway was the main pathway in general cases to induce cell death, but when apoptosis pathway was inhibited, necroptosis pathway could be increased.

In the study, although we found the existence of the necroptotic pathways in osteoblasts cell death induced by breast cancer cells, some research results still need to be improved, including a certain difference between the detected protein and the corresponding mRNA expression. For analysis of the reason that the change of mRNA expression level did not match with their corresponding proteins level, we suggested that this difference may be due to the fact that we detect functional proteins through Western blot, while some non-functional proteins were also expressed through PCR detection. Therefore, in the further study, we need to research the relationship between mRNA and non-functional proteins.

\section{Conclusions}

Our study aims to establish a co-culture system of breast cancer cells and osteoblasts in vitro, so as to achieve the effect of in vivo osteolysis, and investigate whether there is a necroptosis pathway in the osteoblasts death induced by breast cancer cells in vitro. In previous studies have shown that the osteolysis of breast cancer cells mainly due to that breast cancer cells promote the proliferation of osteoclasts and the apoptosis of osteoblasts, which breaks the balance between osteoclast and osteoclast. Our study verified the breast cancer cells can promote osteoblasts apoptosis and also lead to necroptosis of osteoblasts, moreover it provides a new idea and method for the clinical treatment of osteonecrosis caused by bone metastasis of breast cancer, namely the combination of necroptosis inhibitor Nec-1 and apoptosis inhibitor Z-IETD can reduce the mortality of osteoblasts.

\section{Acknowledgments}

Funding: None.

\section{Footnote}

Conflicts of Interest: All authors have completed the ICMJE uniform disclosure form (available at http://dx.doi. org/10.21037/tcr.2019.11.32). The authors have no conflicts of interest to declare.

Ethical Statement: The authors are accountable for all aspects of the work in ensuring that questions related to the accuracy or integrity of any part of the work are appropriately investigated and resolved. This study mainly involves cell experiments, in which all of the cells used are purchased from the National Infrastructure of Cell Line Resource (China), and none of animals or human is involved in this study, so the Ethics Approval is not required. 
Open Access Statement: This is an Open Access article distributed in accordance with the Creative Commons Attribution-NonCommercial-NoDerivs 4.0 International License (CC BY-NC-ND 4.0), which permits the noncommercial replication and distribution of the article with the strict proviso that no changes or edits are made and the original work is properly cited (including links to both the formal publication through the relevant DOI and the license). See: https://creativecommons.org/licenses/by-nc-nd/4.0/.

\section{References}

1. Jemal A, Bray F, Center MM, et al. Global cancer statistics. CA Cancer J Clin 2011;61:69-90.

2. Cai Z, Liu Q. Understanding the Global Cancer Statistics 2018: implications for cancer control. Sci China Life Sci 2019. [Epub ahead of print].

3. Coleman RE. Clinical features of metastatic bone disease and risk of skeletal morbidity. Clin Cancer Res 2006; 12:6243s-6249s.

4. Paget $\mathrm{S}$. The distribution of secondary growths in cancer of the breast. 1889. Cancer Metastasis Rev 1989;8:98-101.

5. Mundy GR. Mechanisms of bone metastasis. Cancer 1997;80:1546-56.

6. Mercer RR, Miyasaka C, Mastro AM. Metastatic breast cancer cells suppress osteoblast adhesion and differentiation. Clin Exp Metastasis 2004;21:427-35.

7. Mastro AM, Gay CV, Welch DR, et al. Breast cancer cells induce osteoblast apoptosis: a possible contributor to bone degradation. J Cell Biochem 2004;91:265-76.

8. Vanden Berghe T, Linkermann A, Jouan-Lanhouet S, et al. Regulated necrosis: the expanding network of nonapoptotic cell death pathways. Nat Rev Mol Cell Biol 2014;15:135-47.

9. Zhang L, Wei J, Ren L, et al. Endosulfan inducing apoptosis and necroptosis through activation RIPK signaling pathway in human umbilical vascular endothelial cells. Environ Sci Pollut Res Int 2017;24:215-25.

10. Newton K, Dugger DL, Wickliffe KE, et al. Activity of

Cite this article as: Ji X, Wang R, Tang $\mathrm{H}$, Chen $\mathrm{H}$, Bao L, Feng F, Jia P. Necroptosis of osteoblasts was induced by breast cancer cells in vitro. Transl Cancer Res 2020;9(2):500-507. doi: $10.21037 /$ tcr.2019.11.32 protein kinase RIPK3 determines whether cells die by necroptosis or apoptosis. Science 2014;343:1357-60.

11. Zhou Y, Dai W, Lin C, et al. Protective effects of necrostatin-1 against concanavalin A-induced acute hepatic injury in mice. Mediators Inflamm 2013;2013:706156.

12. Zhang Y, Zhang J, Yan R, et al. Receptor-interacting protein kinase 3 promotes platelet activation and thrombosis. Proc Natl Acad Sci U S A 2017;114:2964-9.

13. Adameova A, Hrdlicka J, Szobi A, et al. Evidence of necroptosis in hearts subjected to various forms of ischemic insults. Can J Physiol Pharmacol 2017;95:1163-9.

14. Kaczmarek A, Vandenabeele P, Krysko DV. Necroptosis: the release of damage-associated molecular patterns and its physiological relevance. Immunity 2013;38:209-23.

15. Wang T, Jin Y, Yang W, et al. Necroptosis in cancer: An angel or a demon? Tumour Biol 2017;39:1010428317711539.

16. Quayle L, Ottewell PD, Holen I. Bone Metastasis: Molecular Mechanisms Implicated in Tumour Cell Dormancy in Breast and Prostate Cancer. Curr Cancer Drug Targets 2015;15:469-80.

17. Quiroz-Munoz M, Izadmehr S, Arumugam D, et al. Mechanisms of Osteoblastic Bone Metastasis in Prostate Cancer: Role of Prostatic Acid Phosphatase. J Endocr Soc 2019;3:655-64.

18. Galluzzi L, Joza N, Tasdemir E, et al. No death without life: vital functions of apoptotic effectors. Cell Death Differ 2008;15:1113-23.

19. He S, Wang L, Miao L, et al. Receptor interacting protein kinase-3 determines cellular necrotic response to TNFalpha. Cell 2009;137:1100-11.

20. Ji X, Xu F, Dong G, et al. Loading necrostatin-1 composite bone cement inhibits necroptosis of bone tissue in rabbit. Regen Biomater 2019;6:113-9.

21. Koshinuma S, Miyamae M, Kaneda K, et al. Combination of necroptosis and apoptosis inhibition enhances cardioprotection against myocardial ischemia-reperfusion injury. J Anesth 2014;28:235-41. 